\title{
Ultrastructural Preservation of Plasma Membranes by Non-Lethal Slow Freezing to Liquid Nitrogen Temperature
}

\author{
Seizo Fujikawa and Kiyoshi Miura* \\ Institute of Low Temperature Science, and Faculty of Agriculture*, Hokkaido \\ University, Sapporo 060, Japan
}

\begin{abstract}
Secondary hyphae of Lyophyllum ulmarium were shown to tolerate slow freezing, which allowed extracellular freezing, to $-196^{\circ} \mathrm{C}$. A freeze-fracture study showed that under this non-lethal freezing condition, the plasma membrane of the secondary hyphae did not show any ultrastructural changes as compared with the control, except gross cellular shrinkage. Tertiary hyphae of Lyophyllum ulmarium, on the other hand, were completely injured by slow freezing to $-196^{\circ} \mathrm{C}$, and the plasma membrane showed distinct intramembrane particle aggregation as a result of direct membrane contact caused by severe cellular deformation. It is suggested that the absence of freezing injury in the secondary hyphae was due to ultrastructural preservation of the plasma membrane, which resulted from avoidance of severe cellular deformation, while occurrence of freezing injury in the tertiary hyphae is considered to be due to ultrastructural changes in the plasma membrane caused by severe cellular deformation.
\end{abstract}

Biological freezing in nature is caused, in almost all cases, by very slow cooling rates, and it results in extracellular freezing, in which ice crystals are formed extracellularly but not intracellularly (10). This extracellular freezing subjects cells to stresses such as dehydration, exposure to concentrated solutions, and mechanical deformation produced by the growth of extracellular ice crystals $(6,11)$. The magnitude of these freezing stresses increases gradually with the reduction of temperature until around $-30^{\circ} \mathrm{C}$, at which all solutions have solidified (11). Although most biological systems are damaged by being exposed to freezing, some of them can tolerate slow freezing at certain temperatures ranging from $-3^{\circ}$ to $-196^{\circ} \mathrm{C}$ and for certain periods ranging from a day to a year $(1,7,17,19)$. The minimal freezing temperatures which allow survival after thawing depend upon species, phases of growth, and conditions of acclimation $(10,18)$.

Accumulated evidence indicates that the primary site of freezing injury is the plasma membrane because there is a loss in its semipermeability following lethal freezing (18). Although this result is restricted to materials which can tolerate freezing only at near subzero temperatures, recent morphological studies have shown that freezing of cells to lethal temperatures is accompanied by plasma membrane ultrastructural changes such as lamellar-to-hexagonal ${ }_{\text {II }}$ phase transition (8), formation of intramembrane particle (IMP)-free patches (13), and endoplasmic vesiculation (9), while freezing of these cells to non-lethal temperatures does not produce any ultrastructural 
changes in the plasma membrane. In our previous study (6), we also showed that slow freezing to near subzero temperatures around $-10^{\circ} \mathrm{C}$ produced distinct IMP aggregation in tertiary hyphae of Lyophyllum ulmarium. This IMP aggregation took place by direct intrahyphal plasma membrane contact as a result of severe cellular deformation caused by the formation of extracellular ice crystals (6). A close relation was also found between the percent occurrence of freezing injury and the percent of tertiary hyphae showing deformation-induced IMP aggregation (6).

In this study, we examined further whether there was also a relationship between plasma membrane ultrastructure and cellular function in secondary hyphae of Lyophyllum ulmarium, which could tolerate freezing to $-196^{\circ} \mathrm{C}$.

\section{MATERIALS AND METHODS}

Materials. The materials used in this study were secondary hyphae of Lyophyllum ulmarium (Fr.) Kühner, which had been preserved in the Department of Forest Products, Faculty of Agriculture, Hokkaido University. A small amount of preserved hyphae was placed in a sterile flask containing culture fluid $(20 \mathrm{~g}$ starch, $20 \mathrm{~g}$ sucrose, $1.5 \mathrm{~g}$ polypeptone, $3 \mathrm{~g}$ yeast extract, $0.5 \mathrm{~g} \mathrm{MgSO}_{4} \cdot 7 \mathrm{H}_{2} \mathrm{O}, 1 \mathrm{mg} \mathrm{FeCl}, 1 \mathrm{mg} \mathrm{MnSO}_{4}, 0.4 \mathrm{mg} \mathrm{ZnCl}, 0.1 \mathrm{mg}$ $\mathrm{CuSO}_{4}, 0.5 \mathrm{mg} \mathrm{CaCl} \cdot 2 \mathrm{H}_{2} \mathrm{O}$, and $0.5 \mathrm{ml}$ bean oil per liter of $0.01 \mathrm{M}$ sodium phosphate buffer, pH 5.0) and incubated for 6 days at $25^{\circ} \mathrm{C}$. After incubation, the secondary hyphae were centrifuged, washed three times with distilled water, and used.

In this study, the fruit-bodies of Lyophyllum ulmarium, consisting of tertiary hyphae, were also used to compare the effects of freezing. Fresh fruit-bodies of Lyophyllum ulmarium, cultivated at the Agricultural Association in Iiyama, Nagano Prefecture, were purchased from a local market.

Slow freezing of freeze-fracture samples. The washed secondary hyphae were mounted on freeze-fracture holders, kept at $-5^{\circ} \mathrm{C}$, and a small amount of ice was added to initiate freezing. The frozen samples were further cooled to $-50^{\circ} \mathrm{C}$ at a cooling rate of $0.1^{\circ} \mathrm{C} / \mathrm{min}$ by the method previously described $(2,3,6)$. After reaching $-50^{\circ} \mathrm{C}$, they were immersed in liquid nitrogen, because after the samples reached $-50^{\circ} \mathrm{C}$, at which all the solutions had solidified, cellular survival was not affected by the cooling rates (16). This freezing process resulted in extracellular freezing and is referred to as "slow freezing" in this study.

Small pieces $(4 \times 4 \times 6 \mathrm{~mm})$ of fruit-body cap consisting of tertiary hyphae were cut, mounted on freeze-fracture holders, and frozen as the secondary hyphae had been. These samples were stored in liquid nitrogen until use.

Measurement of freezing damage. For measuring freezing damage, about $0.4 \mathrm{~g}$ washed secondary hyphae or a small piece of fruit-body was put into a tube, frozen in the same manner as the freeze-fracture samples, kept for 1 day in liquid nitrogen, and then thawed slowly for $6 \mathrm{~h}$ in a cold chamber kept at $0^{\circ} \mathrm{C}$. Two $\mathrm{ml}$ of distilled water were added to each test tube containing a freeze-thawed sample or an unfrozen control sample. The tubes were vigorously shaken overnight to promote efflux of ions from damaged hyphae. The conductivity of water was read using a conductivity meter (M \& S Instruments Inc., CD-35MII). The test tubes were then boiled for $5 \mathrm{~min}$ and the conductivity of water was read again. The conductivity of water before boiling was calculated as a percentage of that after boiling to give percent leakage. The percent damage was calculated from the following formula (13):

$$
\% \text { damage }=\frac{100(\% \text { leakage } \mathrm{F}-\% \text { leakage } \mathrm{C})}{100-\% \text { leakage } \mathrm{C}},
$$

where $\mathrm{F}$ is the frozen sample, and $\mathrm{C}$ is the unfrozen control sample. 
The growth test was also done on sterile frozen-thawed samples by incubating them in a culture fluid (secondary hyphae) and in a slant culture (tertiary hyphae) at $25^{\circ} \mathrm{C}$ for 6 days.

Electron microscopy. Freeze-fracture replicas were made from slowly frozen samples in the usual way by using a JFD-7000 freeze-fracture device (JEOL Co., Ltd.). The samples were removed from the replicas by $95 \%$ sulfuric acid and commercial bleach. For control, freezereplicas were made from (1) fresh hyphae, which were mounted on a freeze-fracture holder and frozen rapidly from room temperature with Freon 22 cooled by liquid nitrogen, and (2) fresh hyphae, which were fixed with $5 \%$ glutaraldehyde in $50 \mathrm{mM}$ sodium phosphate buffer, $\mathrm{pH} 7.2$, for $5 \mathrm{~h}$ at $4{ }^{\circ} \mathrm{C}$, impregnated with $30 \%$ glycerol, mounted on a holder, and frozen rapidly with Freon 22 . For control, fresh hyphae were fixed with $5 \%$ glutaraldehyde in the same way as the freeze-fracture samples, post-fixed with $2 \%$ osmium tetroxide in distilled water for $2 \mathrm{~h}$ at $4^{\circ} \mathrm{C}$, dehydrated with ethanol, and embedded with Epon 812. Thin sections were cut by a diamond knife mounted in an Ultracut Om U4 (Reichert-Jung), and then stained with $2 \%$ aqueous uranyl acetate and lead citrate. Both freeze-fracture replicas and thin sections were examined by a JEM 100C electron microscope (JEOL Co., Ltd.) operated at an accelerating voltage of $100 \mathrm{KV}$.

The IMP number $/ \mu \mathrm{m}^{2}$ was counted with the naked eye on photographs enlarged to $\mathrm{X}$ 100,000 . The values indicated in the results are means \pm SD of 10 photographs for each treatment.

\section{RESULTS}

\section{SECONDARY HYPHAE}

Post-thawing damage. Post-thawing damage of secondary hyphae slowly frozen to $-196^{\circ} \mathrm{C}$ showed the low rate of $9 \% \pm 7 \%$ (mean $\pm \mathrm{SD}$ from 10 samples) as measured by the leakage of ions. The regrowth of hyphae after freeze-thawing showed a high rate similar to that of unfrozen hyphae. Thus, the majority of secondary hyphae could tolerate slow freezing to $-196^{\circ} \mathrm{C}$.

Ultrastructure of control. The unfrozen secondary hyphae prepared by thin sectioning showed a cytoplasm that included nuclei, vacuoles, electron dense oil droplets, mitochondria, and endoplasmic reticula (Fig. 1, and for the ultrastructure of hyphae, see ref. 12). Figure 2 shows the ultrastructure of control hyphae prepared

Fig. 1. A thin section electron micrograph of a control hyphae. $\times 16,000$.

Fig. 2. A freeze-fracture electron micrograph of a control secondary hyphae prepared by the ordinary method using fixation with glutaraldehyde and glycerin impregnation. PF; protoplasmic face of the plasma membrane. EF; exoplasmic face of the plasma membrane. The arrow indicates the direction of shadowing. $\times 24,000$.

Fig. 3. A freeze-fracture electron micrograph of a secondary hyphae rapidly frozen from room temperature. $\times 24,000$.

Fig. 4. A freeze-fracture electron micrograph of hyphae slowly frozen to $-196^{\circ} \mathrm{C}$. In several hyphae, indicated from $a$ to $l$, agglutination occurred due to the formation of large extracellular ice. Each hyphae was shrunken, but the severe deformation which might cause direct, intrahyphal plasma membrane contact did not take place even in the most deformed regions of the hyphae (arrowheads). $\times 16,500$.

Fig. 5. A freeze-fracture electron micrograph of secondary hyphae slowly frozen to $-196^{\circ} \mathrm{C}$. Each hyphae was shrunken, but no ultrastructural changes of the plasma membrane are seen. $\times 58,000$. 

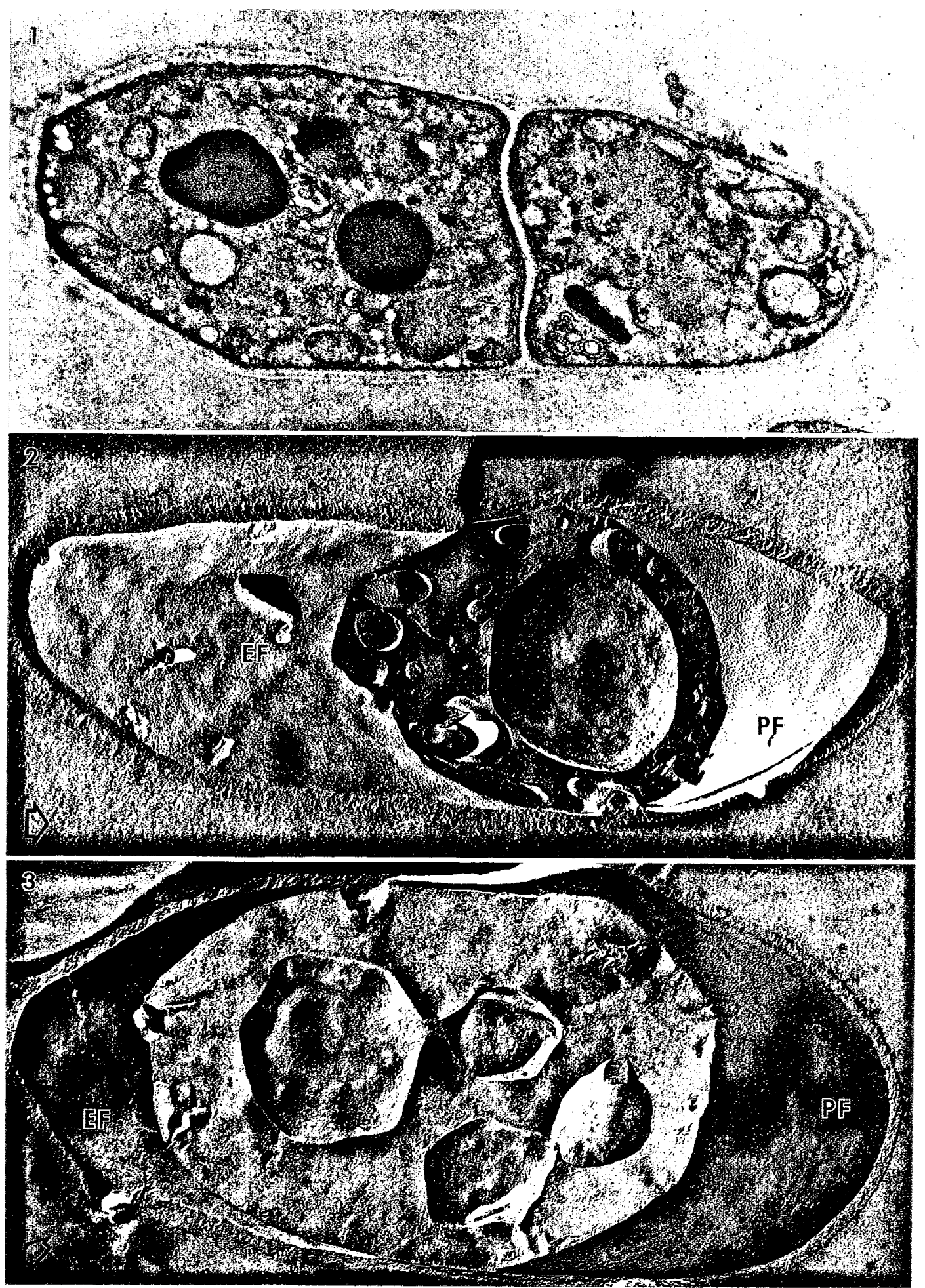

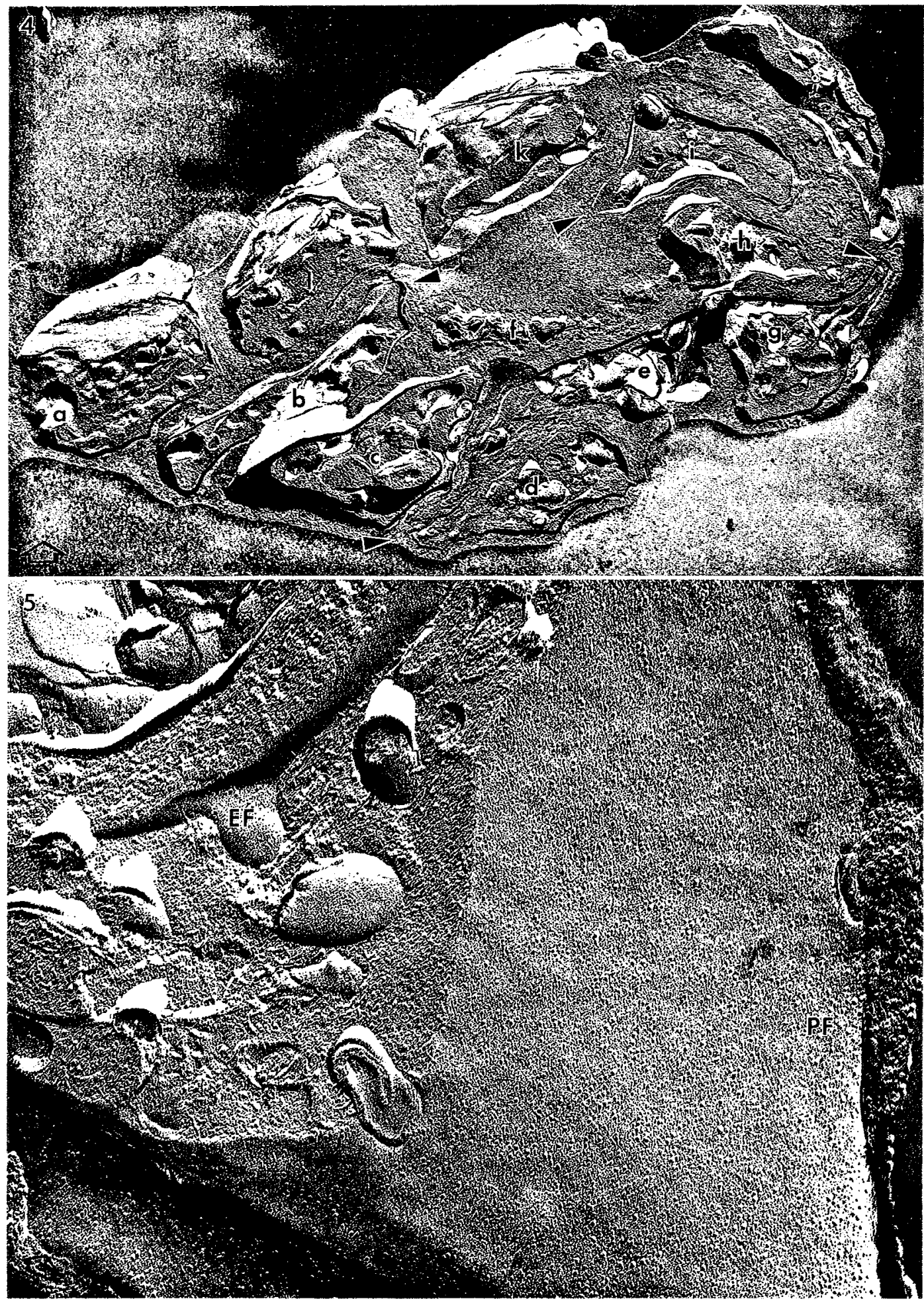
by freeze-fracture using fixation with glutaraldehyde followed by glycerol impregnation. In this ordinary freeze-fracture preparation, the IMPs were distributed evenly through the fracture faces of the plasma membranes. The IMP density of the plasma membrane was $1367 \pm 199 / \mu \mathrm{m}^{2}$ in the protoplasmic face (PF) and $116 \pm 14 / \mu \mathrm{m}^{2}$ in the exoplasmic face (EF). No distinct IMP aggregation was seen in cytoplasmic membranes such as nuclei, vacuoles, and mitochondria.

Figure 3 shows a hyphae rapidly frozen from room temperature. The hyphae was frozen intracellularly by rapid freezing, and ice crystals inside the cell were observed. Similar to the hyphae prepared by thin sectioning (Fig. 1) and ordinary freezefracture (Fig. 2), however, the cross fractured view of the hyphae was almost circular, indicating that little dehydration took place by this rapid freezing. The IMPs on the plasma membrane were evenly distributed by rapid freezing. The IMP density on the plasma membrane was $1164 \pm 112 / \mu \mathrm{m}^{2}$ in PF and $404 \pm 65 / \mu \mathrm{m}^{2}$ in $\mathrm{EF}$, thus clearly differing from ordinary freeze-fracture samples. The cytoplasmic membranes also showed no distinct IMP aggregation by rapid freezing.

Ultrastructure by slow freezing. The secondary hyphae slowly frozen to $-196^{\circ} \mathrm{C}$ were shrunken by extracellular freezing. The hyphae were dehydrated and no intracellular ice crystals were formed (Figs. 4 and 5). Several hyphae were agglutinated by the formation of large extracellular ice during slow freezing. However, gross shrinkage of each hyphae did not result in severe deformation, which might bring about direct intrahyphal contact of plasma membranes by moving the cytoplasm aside. Even in the most deformed regions in the hyphae (arrowheads in Fig. 4), no direct contact of plasma membranes took place. The IMPs on the plasma membrane were distributed evenly and no detectable ultrastructural changes of plasma membranes were observed as compared with the rapidly frozen control samples. The IMP density of the plasma membranes was $1182 \pm 181 / \mu \mathrm{m}^{2}$ in the PF and $389 \pm 58 / \mu \mathrm{m}^{2}$ in the $\mathrm{EF}$, values similar to those of the rapidly frozen control samples. The cytoplasmic membranes also showed no detectable ultrastructural changes due to slow freezing.

\section{TERTIARY HYPHAE}

Post-thawing damage. Post-thawing damage of tertiary hyphae slowly frozen to $-196^{\circ} \mathrm{C}$ showed the high rate of $96 \% \pm 3 \%$ as measured by ion leakage. Furthermore, there was no evidence of regrowth in tertiary hyphae thawed after freezing to $-196^{\circ} \mathrm{C}$

Ultrastructure of control. The control tertiary hyphae prepared by thin sectioning or by ordinary freeze-fracture replicas were almost circular in the cross sectional view, as shown in the previous study (6). The IMPs were evenly distributed through the fracture faces of plasma membrane (6).

Ultrastructure by slow freezing. The tertiary hyphae frozen slowly to $-196^{\circ} \mathrm{C}$ showed severe deformation as a result of extracellular freezing, and a part of the hyphae was held and flattened, which resulted in direct, intrahyphal plasma membrane contact because the cytoplasm was moved aside (Fig. 6). The plasma membranes of these hyphae showed local IMP aggregation (Fig. 7). The membrane regions showing IMP aggregation were strictly restricted to the regions in which the inner surface areas of the plasma membrane were brought into direct contact. The extensive membrane areas without such contact kept an even IMP distribution. After freezing to $-196^{\circ} \mathrm{C}, 100 \%$ of the tertiary hyphae $(\mathrm{n}=100)$ showed plasma membranes with deformation-induced IMP aggregation. 

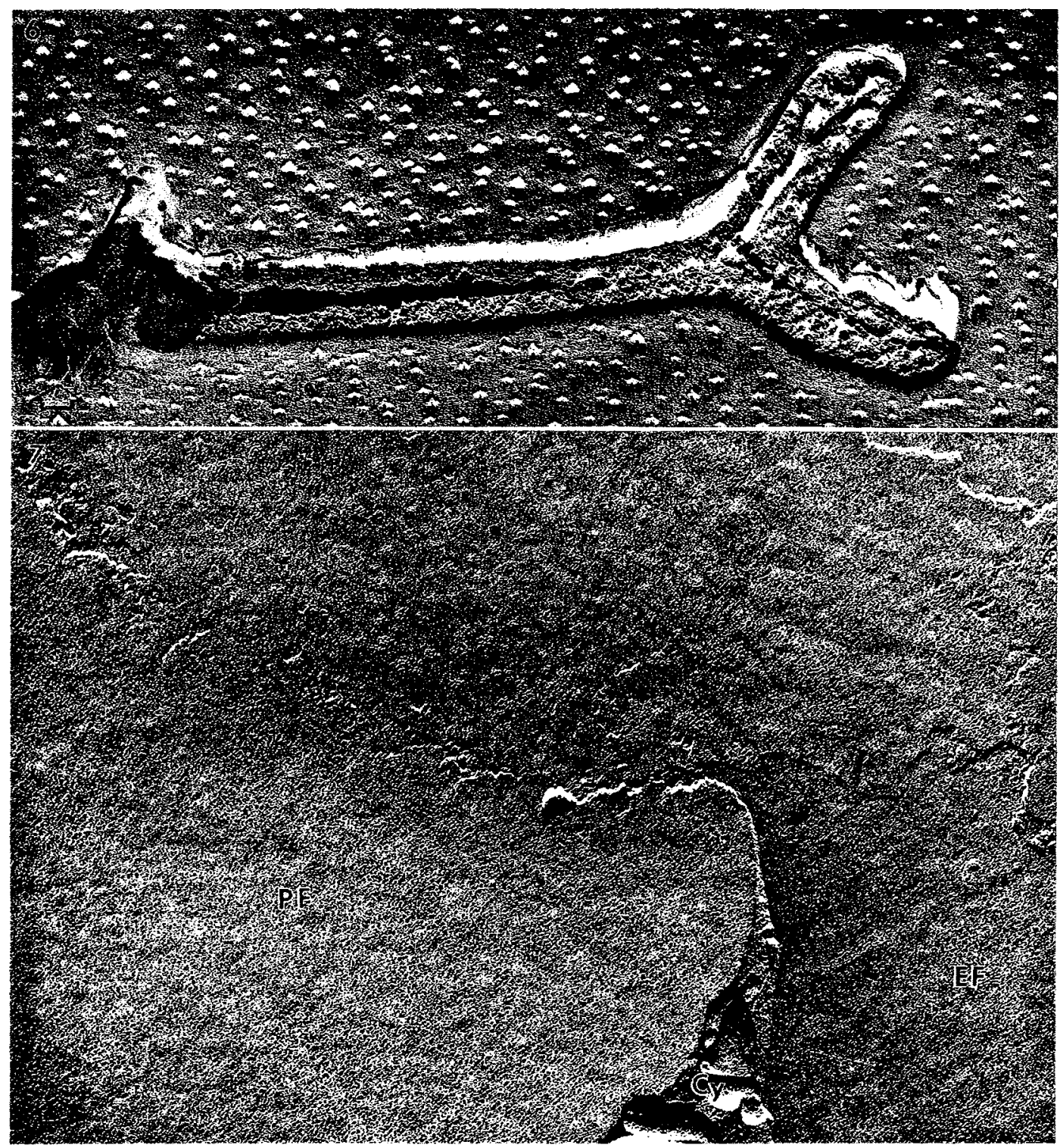

Fig. 6. A freeze-fracture electron micrograph of a tertiary hyphae slowly frozen to $-196^{\circ} \mathrm{C}$. The hyphae was severely deformed and the plasma membranes were brought into direct contact. $\times 32,000$.

Fig. 7. A freeze-fracture electron micrograph of a part of a tertiary hyphae slowly frozen to $-196^{\circ} \mathrm{C}$. The IMPs are distinctly aggregated in the plasma membrane regions where the membranes have been brought into direct contact (the upper half of the figure), but not in the plasma membrane regions without such contact (the lower half of the figure). Cy; cytoplasm. $\times 33,000$. 


\section{DISCUSSION}

Secondary hyphae of Lyophyllum ulmarium could tolerate slow freezing to $-196^{\circ} \mathrm{C}$, and except for gross cellular shrinkage, no discernible ultrastructural changes of the plasma membranes were found as compared with the control. The IMP density and distribution of the plasma membranes of slowly frozen secondary hyphae were similar to those of the rapidly frozen control, although the rapid freezing used in this study was estimated to be a rate of $8,000^{\circ} \mathrm{C} / \mathrm{min}$, which was slower than the cooling rate which results in a vitreous state (3). Freezing stresses, especially at the slow cooling rates, may cause change of IMP distribution in other cell types (5). The fact that there was no discernible difference in IMP density and distribution between freezing at $0.1^{\circ}$ and $8,000^{\circ} \mathrm{C} / \mathrm{min}$, however, implies that the IMP distribution of secondary hyphae is insensitive to cooling rates over a wide range. Thus, the rapid freezing done in this study seemed to be adequate to fix IMPs without effecting freezing stresses. A difference in IMP density was found only between the chemically fixed and unfixed samples. This difference may be an inherent one between the chemically fixed and unfixed samples in a variety of cell types $(14,20)$. It is suggested that the different densities of IMPs between chemically fixed and unfixed samples were responsible for the different degrees of stability of IMPs upon freeze-fracturing (4).

Recent morphological studies have shown that slow freezing stresses bring about some ultrastructural changes of plasma membranes in association with injury, while non-lethal freezing does not cause ultrastructural changes of plasma membranes $(8,9,13)$. Pearce and Willison (13) have shown that slow freezing produces IMP-free patches on the plasma membrane in wheat seedlings only by freezing to below $-10^{\circ} \mathrm{C}$, at which temperature freezing injury takes place. It is suggested that the IMP-free patches form due to dehydration during slow freezing (13). Gordon-Kamm and Steponkus (8) have shown that upon slow freezing to $-10^{\circ} \mathrm{C}$, non-acclimated rye protoplasts suffer severe freezing injury in association with the formation of the hexagonal ${ }_{11}$ phase in the plasma membranes as a result of cellular dehydration during freezing, while the cold-acclimated rye protoplasts which can tolerate freezing to at least $-10^{\circ} \mathrm{C}$ maintain ultrastructural integrity in the plasma membranes in spite of being frozen to $-10^{\circ} \mathrm{C}$. They suggested that the different response betweenacclimated and non-acclimated rye protoplasts against freezing stresses is due to the different nature of their plasma membrane constituents. A chemical analysis of the membrane constituents in relation to freezing tolerance will be done in near future by a method which extracts highly purified plasma membrane fractions from plant cells (21).

In our previous paper (6), we showed that in the tertiary hyphae of Lyophyllum ulmarium, extensive plasma membrane areas kept ultrastructural integrity during slow freezing to $-30^{\circ} \mathrm{C}$, except for membrane regions with direct contact. We suggested that the plasma membrane was stable against dehydration and exposure to concentra ted solutions during slow freezing to at least $-30^{\circ} \mathrm{C}(6)$. In those samples, however, freezing injury occurred as early as in slow freezing to $-5^{\circ} \mathrm{C}(6)$. The occurrence of this injury was related to the formation of IMP aggregation in the plasma membranes, which took place by the direct, intrahyphal contact of plasma membranes as a result of severe mechanical deformation caused by formation of extracellular ice (6). In the present study, it was also shown that slow freezing of tertiary hyphae to $-196^{\circ} \mathrm{C}$ resulted in almost complete injury, and a corresponding number of hyphae showed 
deformation-induced IMP aggregation. It is suggested that this IMP aggregation was caused by mutual electrostatic repulsion between contacting membranes and that such contact resulted in membrane fusion (6) and/or lamellar-to-hexagonal ${ }_{\text {II }}$ phase transition during thawing (15), which might lead to injury.

In the secondary hyphae, freezing to $-196^{\circ} \mathrm{C}$ did not cause direct contact of plasma membrane. Consequently, no deformation-induced IMP aggregation was produced, and the plasma membranes retained their ultrastructural integrity. The present study thus shows that the ultrastructural preservation of plasma membranes is closely related to the functional preservation of the frozen cells, not only to near subzero temperatures but also to liquid nitrogen temperature. The absence of severe cellular deformation is considered to be due mainly to a higher concentration of cytoplasmic substances or to a lower content of intracellular water in the secondary hyphae. Thus the prevention of slow freezing injury in secondary hyphae is greatly dependent on the nature of the cytoplasm as well as on the nature of the plasma membranes, which are resistant to dehydration and exposure to concentrated solutions.

\section{REFERENCES}

1. Asahina, E. Freezing and frost resistance in insects. in Cryobiology, ed. Meryman, H. T., Academic Press, London and New York, pp. 451-486, 1966

2. Fusikawa, S. The effect of various cooling rates on the membrane ultrastructure of frozen human erythrocytes. in Effects of Low Temperatures on Biological Membranes, ed. MoRRIs, G.J., and A. Clarke, Academic Press, London, pp. 323-334, 1981

3. FujiKaWA, S. The effects of various cooling rates on the membrane ultrastructure of frozen human erythrocytes and its relation to the extent of haemolysis after thawing. J. Cell Sci. 49, 369-382, 1981

4. Fujikawa, S. Difference of intramembraneous particle fracture between glutaraldehyde-fixed and -unfixed erythrocyte membranes as revealed by the negative staining carbon replica method. J. Electron Microsc. 31, 303, 1982

5. Fujikawa, S. Freeze-etching study on membrane ultrastructural changes caused by freezing: cooling rate-dependent intramembrane particle aggregation in erythrocyte stripped ghosts. Cryobiology 22, 60-76, 1085

6. Fujikawa, S. and K. MiURa. Plasma membrane ultrastructural changes caused by mechanical stress in the formation of extracellular ice as a primary cause of slow freezing injury in fruitbodies of Basidiomycetes (Lyophyllum ulmarium (Fr.) Kühner). Cryobiology 23, 371-382, 1986

7. Fujikawa, S. and K. Miura. Freezing tolerance in edible mushrooms. Jpn. J. Freezing and Drying 32, 14-17, 1986

8. Gordon-Kamm, W.J. and P.L. STEPonkus. Lamellar-to-hexagonal ${ }_{I I}$ phase transition in the plasma membrane of isolated protoplasts after freeze-induced dehydration. Proc. Natl. Acad. Sci. USA. 81, 6373-6377, 1984

9. Gordon-KAmm, W.J. and P.L. Steponkus. The influence of cold acclimation on the behavior of the plasma membrane following osmotic contraction of isolated protoplasts. Protoplasma 123, 161-173, 1984

10. Levitt, J. Responses of Plants to Environmental Stresses. Chilling, Freezing, and High Temperature Stresses: Physiological Ecology, Vol. 1, 2nd Ed., Academic Press, New York, pp. 1-290, 1980

11. MAZUR, P. Physical and chemical basis of injury in single-celled micro-organisms subjected to freezing and thawing. in Cryobiology, ed. Meryman, H.T., Academic Press, London and New York, pp. 213-315, 1966

12. Moore, R.T. The ultrastructure of fungal cell. in The Fungi, I. The Fungal Cell, ed. 
Ainsworth, G.C., and A.S. Sussman, Academic Press, New York and London, pp. 95-118, 1965

13. Pearce, R.S. and J.H.M. Willison. A freeze-etch study of the effects of extracellular freezing on cellular membranes of wheat. Planta 163, 304-316, 1985

14. Pricam, C., K.A. Fisher and D.S. Friend. Intramembranous particle distribution in human erythrocytes: effects of lysis, glutaraldehyde, and poly-L-lysine. Anat. Rec. 189, 295-308, 1985

15. QuinN, P.J. A lipid-phase separation model of low-temperature damage to biological membranes. Cryobiology 22, 128-146, 1985

16. SAKaI, A. Survival of plant tissue at super-low temperatures III. Relation between effective prefreezing temperatures and the degree of frost hardiness. Plant Physiol. 40, 882-887, 1965

17. SAKAI, A. Studies of frost hardiness in woody plants II. Effects of temperature on hardening. Plant Physiol. 41, 353-359, 1966

18. Steponkus, P.L. Role of the plasma membrane in freezing injury and cold acclimation. Annu. Rev. Plant Physiol. 35, 543-584, 1984

19. Storey, K.B. Freeze tolerance in terrestrial frogs. Cryo Lett. 6, 115-134, 1985

20. Willison, J.H.M. and R.M. Brown, Jr. Pretreatment artifacts in plant cells. in Freeze Fracture: Methods, Artifacts, and Interpretations, ed. RASH, J. E., and C. S. Hudson, Raven Press, New York, pp. 51-57, 1981

21. Yoshida, S., M. Uemura, T. Niki, A. Sakai and L.V. Gusta. Partition of membrane particles in aqueous two-polymer phase system and its practical use for purification of plasma membrane from plants. Plant Physiol. 72, 105-114, 1983

(Received for publication, September 24, 1986) 